\section{Hyperactive antifreeze protein from beetles}

$\bigvee_{\text {e have purified a thermal hysteresis }}$ (antifreeze) protein, with up to 100 proteins, from the common yellow mealworm beetle, Tenebrio molitor. It is a threonine- and cysteine-rich protein, of relative molecular mass 8,400, composed largely of 12 -amino-acid repeats. We estimate that a concentration of roughly $1 \mathrm{mg} \mathrm{ml}^{-1}$ of this protein can account for the $5.5^{\circ} \mathrm{C}$ of thermal hysteresis found in Tenebrio larvae (Fig. 1).

Although protein-mediated thermal hysteresis was noted in Tenebrio almost 30 years ago ${ }^{1}$, numerous attempts to purify any thermal hysteresis proteins (THPs) failed to yield activities (quantified as the temperature difference between the freezing and melting points of a solution containing ice) sufficient to account for that of haemolymph $^{1-5}$. We have now isolated microgram quantities of THP from diluted larval Tenebrio haemolymph by gel exclusion chromatography followed by reversedphase high-performance liquid chromatography (HPLC) on an analytical C18 column. Neighbouring HPLC fractions corresponded to discrete proteins (THP26 and THP27). The thermal hysteresis activity of THP26 at $55 \mu \mathrm{g} \mathrm{ml}^{-1}$ was $1.6{ }^{\circ} \mathrm{C}$ and of THP27 at $21 \mu \mathrm{g} \mathrm{ml}^{-1}$ was $1.1^{\circ} \mathrm{C}$, close to the maximum values obtained with 10-30 $\mathrm{mg} \mathrm{ml}^{-1}$ of fish antifreeze proteins (AFPs) ${ }^{6}$.

Because the amino termini of THP26 and THP27 were blocked, we sequenced an internal fragment from THP26 that was released by endoproteinase Lys- $\mathrm{C}$ digestion (Fig. 2). We used degenerate and vector primers in the polymerase chain reaction and screened a Tenebrio complementary DNA library with the product. We obtained the complete coding sequences of four THP variants (YL1-4), whose conceptual translations match the sequenced peptide fragment at up to 18 of the 20 residues (Fig. 2). The first 28 amino acids represent a secretory signal peptide. The $\mathrm{N}$-terminal amino acid of three of the variants is predicted to be glutamine, consistent with N-terminal blockage in which the $\mathrm{N}$-terminal glutamine is converted to pyroglutamate by cyclization. The cleavage site of the fourth variant is not clearly predicted.

The amino-acid compositions of the purified proteins and the deduced mature sequences are very similar. The THPs are particularly rich in cysteine (18-19\%), threonine (21-26\%) and other amino acids with short side chains (glycine, alanine, serine), and are deficient in several hydrophobic amino acids, notably leucine and isoleucine.
The overall hydrophilicity is roughly $55 \%{ }^{7}$, much higher than that of fish AFPs .

The primary structure of the proteins (Fig. 2) is very unusual and unlike any previously known sequence. The first 21 amino acids contain six cysteine residues spaced at irregular intervals $\left(\mathrm{Cx}_{5} \mathrm{Cx}_{2} \mathrm{Cx}_{3} \mathrm{Cx}_{2} \mathrm{Cx}_{2} \mathrm{C}\right)$, and this sequence overlaps with the first of a series of 12-amino-acid repeats, in which cysteine is repeated at six-residue intervals, that continue until the end of the protein. The consensus of these repeats is CTxSxxCxxAxT. The THP variants differ in the number of these repeats. Additional 12amino-acid units appear as insertions in the longer variants, indicating that each may form a functional domain and that the exact order of the repeats might not be crucial for function.

We expressed the shortest of the four cDNAs with a conventional signal cleavage site (YL1) in Escherichia coli (Fig. 2). We detected thermal hysteresis in the supernatant of the cell lysate, indicating that some of the protein was able to fold into an active state. Partially purified recombinant

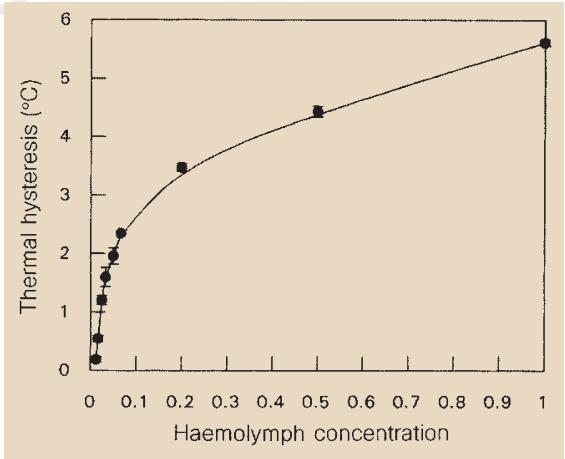

Figure 1 Thermal hysteresis activity of Tenebrio haemolymph as a function of dilution. The standard deviation of each sample $(n=3)$ is shown.

Figure 2 Predicted amino-acid sequences of four THP variants obtained from a fat-body cDNA library. The predicted $\mathrm{N}$-terminal residues $(+1)$ are denoted by an arrow with the signal peptides shown in italics. The repetitive 12-amino-acid domains are numbered consecutively. Conserved residues found in almost all repeats are indicated in bold. The aminoacid sequence from a purified THP fragment (THP26) is shown for comparison. The first Lys residue is assumed as the protein was cleaved with endo-Lys-C. Undetermined positions were assumed to be Cys. The two amino-acid regions used to design the nested degenerate PCR primers are underlined. Databank accession numbers for the THP sequences YL1-4 are AF010329, AF010330, AF010331 and AF010332, respectively.
THP showed thermal hysteresis of $5.3^{\circ} \mathrm{C}$ and its properties were indistinguishable from the THP in Tenebrio haemolymph. Ice crystals that formed in the presence of haemolymph and recombinant protein were identical (Fig. 3 a,b). This and other THP isoforms could account for all thermal hysteresis activity in the insect, because THP in vitro does not need other factors to attain values of more than $5{ }^{\circ} \mathrm{C}$.

Like fish AFPs, Tenebrio THP seems to act by an adsorption-inhibition mechanism, as ice crystals did not grow until the nonequilibrium freezing point was exceeded. Further, the relationship between thermal hysteresis and THP concentration is hyperbolic (Fig. 1) and qualitatively indistinguishable from that of fish AFPs?. However, ice crystals formed in the presence of THPs

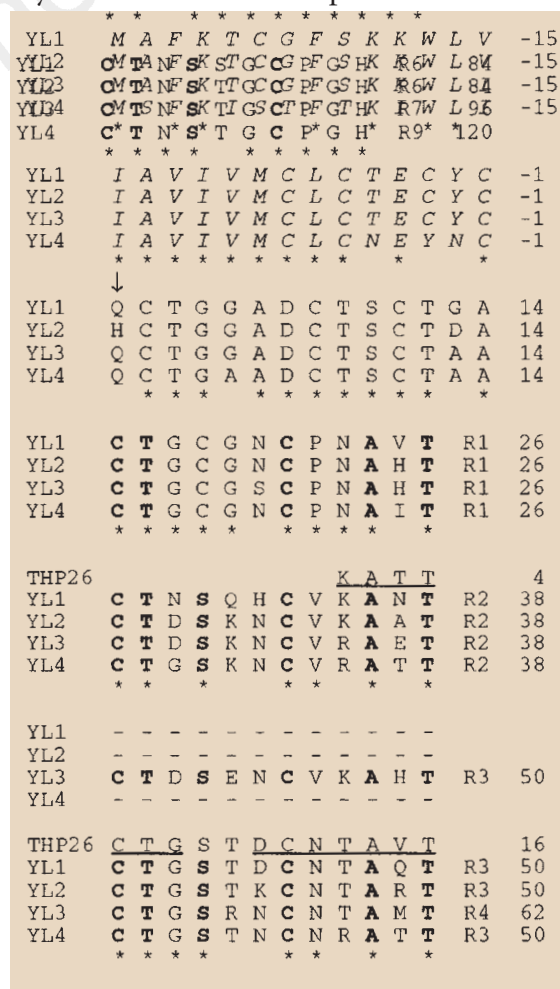
THP26 C T N S K 21 YL1 C C T N S K D C F E A N T R4 62

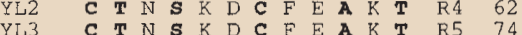
$\begin{array}{llllllllllllll}Y L 4 & C & T & N & S & K & G & C & L & E & A & T & T & R\end{array}$

YL1 YL2
YL3 YL4 


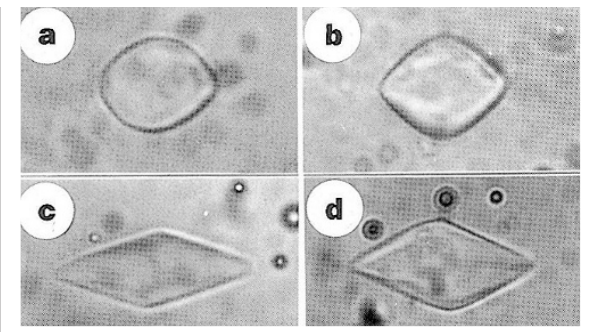

Figure 3 Photomicrographs of ice crystals grown in the presence of THP or AFP. The $c$-axis is horizontal in the plane of the page in all cases. a, Dilute haemolymph. b, Recombinant THP. c, Typet fish AFP. d, Type-ll fish AFP.

were unusual in that their surfaces were curved, whereas crystals generated by fish AFP types I and III are hexagonal bipyramids with flat, well-defined facets (Fig. 3 $\mathrm{c}, \mathrm{d})$, reflecting, in the case of type-I AFP, affinity for a specific pyramidal plane ${ }^{10}$.

The maximum observed thermal hysteresis value $\left(5.5^{\circ} \mathrm{C}\right)$ is four times that obtained with fish AFPs ${ }^{6}$. At low concentrations the activity is up to 100 times that of fish AFPs, consistent with our observations that THP is a minor constituent of haemolymph. Contamination with as little as $0.1-1 \%$ THP could produce activities comparable to those obtained with fish AFPs, suggesting that past THP preparations $^{2-5}$ may have been impure.

A possible explanation for the hyperactivity of THP is that the protein has multiple ice-binding sites and that these sites either recognize several different features of ice, or a repeat expressed in several directions on the ice lattice. These properties could increase the frequency of THP binding to ice and might account for the curved ice crystal surfaces. The fourfold increase in maximum thermal hysteresis values might result from closer spacing of THP on the ice surface ${ }^{11}$. The exceptional activity of Tenebrio THP makes it an attractive reagent for applications requiring freeze resistance or the control of ice growth and morphology.

\section{Laurie A. Graham, Yih-Cherng Liou}

Virginia K. Walker, Peter L. Davies

Departments of Biochemistry and Biology,

Queen's University, Kingston,

Ontario K7L 1N6, Canada

e-mail:daviesp@post.queensu.ca

1. Grimstone, A. V., Mullinger, A. M. \& Ramsay, J. A. Phil. Trans. R. Soc. Lond. B 253, 343-382 (1968).

2. Schneppenheim, R. \& Theede, H. Comp. Biochem. Physiol. B 67, 561-568 (1980).

3. Tomchaney, A. P. et al. Biochemistry 21, 716-721 (1982).

4. Patterson, J. L. \& Duman, J. G. J. Exp. Zool. 219, 381-384 (1982).

5. Horwath, K. L., Easton, C. M., Poggioli, G. J., Myers K. \& Schnorr, I. L. Eur. J. Entomol. 93, 419-433 (1996).

6. Davies, P. L. \& Hew, C. L. FASEB J. 4, 2460-2468 (1990).

7. Wishart, D. S. et al. Comput. Appl. Biosci. 10, 121-132 (1994).

8. Sönnichsen, F. D., Sykes, B. D. \& Davies, P. L. Prot. Sci. 4, 460-471 (1995).

9. DeVries, A. L. Annu. Rev. Physiol. 45, 245-260 (1983).

10. Knight, C. A., Cheng, C. C. \& DeVries, A. L. Biophys. J. 59, 409-418 (1991).

11. Wilson, P. W. Cryo-Letters 14, 31-36 (1993).

\section{Role of CED-4 in the activation of CED-3}

Genetic analyses of the nematode Caenorhabditis elegans have identified three core components of the cell-death apparatus ${ }^{1}$. CED-3 and CED-4 promote, whereas CED9 inhibits cell death. Recent studies indicate that CED-4 might interact independently with CED-3 and CED-9, forming the crux of a multicomponent death complex ${ }^{2}$. But except for its role as an adaptor molecule, little is known about CED-4 function. A clue came with the observation that mutation of the phosphate-binding loop (Ploop) of CED-4 disrupts its ability to induce chromatin condensation in yeast ${ }^{3}$. Further, a P-loop mutant of CED-4 (CED-4 ${ }^{\mathrm{K} 165 \mathrm{R}}$ ) fails to process CED-3 in vivo, both in insect $^{4}$ and mammalian cells (unpublished). We now confirm that CED-4 induces CED3 activation and subsequent apoptosis, and that the process requires binding of ATP.

To test whether CED- 4 could bind ATP, we used ATP analogues that label the nucleotide-binding sites on proteins. One such analogue is 5'-fluorosulphonylbenzoyladenosine (FSBA $)^{5,6}$. The natural nucleotide ATP (with $\mathrm{Mg}^{2+}$ ), effectively inhibited FSBA incorporation whereas CTP (with $\mathrm{Mg}^{2+}$ ) did not (Fig. 1a), showing that FSBA binds specifically to CED-4. The photoaffinity ATP analogue 8-azidoadenosine-5' -triphosphate $\left[\alpha-{ }^{32} \mathrm{P}\right]\left(8 \mathrm{~N}_{3}-\mathrm{ATP}\right)$ has also been used to identify ATP-binding proteins $^{7,8}$. As predicted, CED-4 bound $8 \mathrm{~N}_{3}$ ATP and this photoaffinity labelling was attenuated with ATP and $\mathrm{MgCl}_{2}$ (Fig. 1b). Unlike wild-type CED-4, a form of CED-4 (residues 171-549) in which the P-loop motif was deleted (CED-4 $\Delta \mathrm{PL})$, failed to bind the azido analogue (Fig. 1c).

We next assessed the function of the CED-4 P-loop, and tested whether CED-4 activates CED-3 in vitro. We prepared
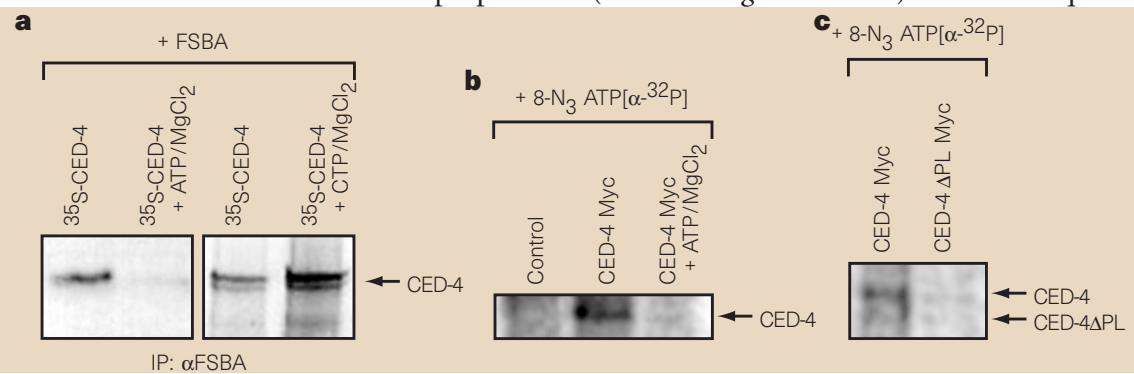

Figure 1 CED-4 binds ATP analogues. a, CED-4 binds FSBA, an inhibitor of P-loop-containing ATPases. ${ }^{35}$ Slabelled CED-4 was incubated with $1 \mathrm{mM}$ FSBA in DMSO in the presence or absence of excess ATP or CTP (10 $\mathrm{mM}$ ) and $\mathrm{MgCl}_{2}(10 \mathrm{mM})$, then immunoprecipitated with rabbit antiFSBA (Boehringer), followed by SDS-PAGE. b, CED-4 binds an azido derivative of ATP. Lysates from 293T cells expressing Myc-CED-4 were incubated with $10 \mu \mathrm{M}$ (final concentration) 8 $\mathrm{N}_{3}$-ATP in the presence or absence of excess ATP $(1 \mathrm{mM})$ and $\mathrm{MgCl}_{2}(1 \mathrm{mM})$. For azido-affinity labelling, samples were irradiated ${ }^{7}$, and then immunoprecipitated with antiMyc antibodies (Boehringer) followed by SDS-PAGE and phosphorimager analysis. Control represents lysates from vector-transfected cells. c, A P-loop deletion mutant of CED-4 fails to bind an azido derivative of ATP. Samples processed as in $\mathbf{b}$. Expression of CED-4 and CED-4APL was confirmed by immunoblotting. 